OPEN ACCESS

Edited by:

Jae Kun Shim,

University of Maryland, College Park,

USA

Reviewed by:

Brian S. Baum,

Regis University, USA

Pedro Figueiredo,

University of Maryland, College Park,

USA

${ }^{*}$ Correspondence:

Julie Stang

julie.stang@nih.no

Received: 09 October 2015

Accepted: 21 January 2016

Published: 05 February 2016

Citation:

Stang J, Wiig H, Hermansen M and

Hansen EA (2016) Voluntary

Movement Frequencies in

Submaximal One- and Two-Legged

Knee Extension Exercise

and Pedaling.

Front. Hum. Neurosci. 10:36.

doi: 10.3389/fnhum.2016.00036

\section{Voluntary Movement Frequencies in Submaximal One- and Two-Legged Knee Extension Exercise and Pedaling}

\author{
Julie Stang ${ }^{1 *}$, Håvard Wiig $^{2}$, Marte Hermansen ${ }^{2}$ and Ernst Albin Hansen ${ }^{3}$ \\ ${ }^{1}$ Department of Sports Medicine, Norwegian School of Sport Sciences, Oslo, Norway, ${ }^{2}$ Department of Physical \\ Performance, Norwegian School of Sport Sciences, Oslo, Norway, ${ }^{3}$ Research Interest Group of Physical Activity and Human \\ Performance, SMI ${ }^{\circledR}$, Department of Health Science and Technology, Aalborg University, Aalborg, Denmark
}

Understanding of behavior and control of human voluntary rhythmic stereotyped leg movements is useful in work to improve performance, function, and rehabilitation of exercising, healthy, and injured humans. The present study aimed at adding to the existing understanding within this field. To pursue the aim, correlations between freely chosen movement frequencies in relatively simple, single-joint, one- and two-legged knee extension exercise were investigated. The same was done for more complex, multiple-joint, one- and two-legged pedaling. These particular activities were chosen because they could be considered related to some extent, as they shared a key aspect of knee extension, and because they at the same time were different. The activities were performed at submaximal intensities, by healthy individuals ( $n=16$, thereof eight women; $23.4 \pm 2.7$ years; $1.70 \pm 0.11 \mathrm{~m}$; $68.6 \pm 11.2 \mathrm{~kg}$ ). High and fair correlations ( $R$-values of 0.99 and 0.75 ) occurred between frequencies generated with the dominant leg and the nondominant leg during knee extension exercise and pedaling, respectively. Fair to high correlations ( $R$-values between 0.71 and 0.95 ) occurred between frequencies performed with each of the two legs in an activity, and the two-legged frequency performed in the same type of activity. In general, the correlations were higher for knee extension exercise than for pedaling. Correlations between knee extension and pedaling frequencies were of modest occurrence. The correlations between movement frequencies generated separately by each of the legs might be interpreted to support the following working hypothesis, which was based on existing literature. It is likely that involved central pattern generators (CPGs) of the two legs share a common frequency generator or that separate frequency generators of each leg are attuned via interneuronal connections. Further, activity type appeared to be relevant. Thus, the apparent common rhythmogenesis for the two legs appeared to be stronger for the relatively simple single-joint activity of knee extension exercise as compared to the more complex multi-joint activity of pedaling. Finally, it appeared that the shared aspect of knee extension in the related types of activities of knee extension exercise and pedaling was insufficient to cause obvious correlations between generated movement frequencies in the two types of activities.

Keywords: cycling cadence, motor control, preferred pedaling rate, rhythmic movement, voluntary motor behavior 


\section{INTRODUCTION}

Better understanding of behavior and control of common voluntary human rhythmic movements is useful in the work to improve performance, function, and rehabilitation of exercising, healthy, and injured humans. Examples of human voluntary stereotyped rhythmic leg movements such as walking (Shapkova, 2004; Minassian et al., 2007), running (Cappellini et al., 2006; Sardroodian et al., 2015), and pedaling (Zehr et al., 2007; Hansen et al., 2014) are widely considered to be controlled by spinal neural networks, termed central pattern generators (CPGs). These CPGs are assisted by tonic supraspinal input and afferent feedback (for review, see previous publications Zehr and Duysens, 2004; Prochazka and Ellaway, 2012). In brief, the internal organization of a CPG is considered to be functionally separated into two components. One of the components is responsible for generation of a rhythmic movement frequency while another is responsible for a rhythmic movement pattern (Perret and Cabelguen, 1980; Kriellaars et al., 1994; McCrea and Rybak, 2008; Dominici et al., 2011). It is further considered that there are separate CPGs for each of the two legs (Shapkova, 2004; Zehr, 2005). Though, many details remain unknown (Ivanenko et al., 2006; Daun-Gruhn, 2011; Hansen, 2015). For example, our knowledge is limited on how common the CPG-generated rhythmogenesis is for man's two legs when considering one- and two-legged systems. Studies of CPG-generated voluntary rhythmic movement in healthy humans are obviously challenged by researchers' limited access to the spinal cord, as compared with studies on animals. Animal studies enable both stimulation of CPGs, measurement of CPG activity, and measurement of behavior. Though, in order to be able to further develop these considerations, analyses of human rhythmic movement behavior may be applied (Goulding, 2009; Schlinger, 2015), which is what was done in the present study.

The overall aim of the present study was to add to the understanding of movement behavior and control in human voluntary stereotyped rhythmic leg movements. In order to meet this objective, correlations between freely chosen movement frequencies in relatively simple, single-joint, one- and two-legged knee extension exercise were investigated. The same was done for more complex, multiple-joint, one- and two-legged pedaling. In case that the present study results in high correlations between movement frequencies generated separately by each of the legs it might be interpreted to support a working hypothesis of the two legs' CPGs to share a common frequency generator, or alternatively, of separate frequency generators of each of the legs to be attuned via interneurons. The present involvement of two related, yet different, types of activities of knee extension exercise and pedaling also allowed the investigation of the following two test hypotheses: (i) The degree of common frequency generation for the two legs (i.e., the correlation coefficients of correlations between frequencies for the legs) is similar in the activity of knee extension exercise and the activity of pedaling. (ii) The shared aspect of knee extension, in itself, in the related types of activities of knee extension exercise and pedaling is sufficient for the generated movement frequencies in the two types of activities to correlate at least moderately. Knee extension contributes to characterize the down stroke phase in pedaling where most of the external power is produced (So et al., 2005).

\section{MATERIALS AND METHODS}

\section{Participants}

Sixteen healthy individuals ( 8 women, 8 men; $23.4 \pm 2.7$ years; $1.70 \pm 0.11 \mathrm{~m} ; 68.6 \pm 11.2 \mathrm{~kg}$ ) volunteered to participate in the study. They were carefully informed about the procedures of the study and the overall aim ("to enlarge our knowledge on control of rhythmic movement") but at the same time kept naive to the specific purpose. The latter served to avoid any particular conscious movement control during the applied activities. All participants occasionally performed cycling as personal transport or exercise, but none of them were competitive cyclists. Fourteen of the participants reported that the right leg was their dominant leg while two participants reported that the left leg was their dominant leg. Written informed consent was obtained from all participants. The present study was carried out in accordance with the recommendations of the regional committee of Southern Norway for research ethics and the study conformed to the standards set by the Declaration of Helsinki.

\section{Test Session}

Each participant was informed to abstain from alcohol and tobacco $24 \mathrm{~h}$ before the test session. In addition, the participant was informed not to perform any intensive exercise $48 \mathrm{~h}$ before the test session.

Each participant reported to the laboratory for a single test session that lasted $1 \mathrm{~h}$ and consisted of the following. First, the participant was allowed to adjust the cycle ergometer with respect to seat and handlebar position. Second, warm up and familiarization to the knee extension activities were performed in form of 5 min of two-legged knee extension exercise followed by $3 \mathrm{~min}$ of rest and $5 \mathrm{~min}$ of one-legged knee extension exercise. After another $3 \mathrm{~min}$ of rest, six activities were performed in random order as 5 min-bouts interrupted by 3 min-rest periods. The six activities consisted of one- and two-legged knee extension exercise as well as one- and two-legged pedaling. The two-legged knee extension exercise was performed in a way that each of the legs was extended in an alternating way as in pedaling. Each participant was informed in "not thinking of anything special" and "letting the legs move by themselves" during the activities. Frequency was freely chosen in all activities. During the activities, the participant was blinded to the frequency and the time.

For knee extension exercise, a custom build electromagnetically braked ergometer was applied (Hallén et al., 1996). In brief, the ergometer consisted of a car seat placed above an electromagnetically braked cycle ergometer, which had aluminum rods mounted on the crank arms instead of pedals. The rods pointed forwards with respect to the seated participant. Braces were attached to the other ends of the rods. The participants' feet were placed in the braces. The knee extension ergometer was constructed in a way that the flywheel only could 
be pulled, by knee extension, and not pushed, by knee flexion. Further, the power output that was delivered to the ergometer was a linear function of flywheel speed. For the present study, the ergometer was programmed for a power output (in W) during one-legged knee extension that corresponded to body mass times 0.5 and 0.6 for women and men, respectively, at a frequency of $60 \mathrm{rpm}$. Power output during two-legged knee extension exercise was twice as high. The construction of the ergometer resulted in power output to be higher or lower if the participants chose a higher or lower, respectively, frequency than $60 \mathrm{rpm}$ during knee extension exercise. The participant's torso was strapped to the seat during knee extension. For pedaling, an electromagnetically braked Excalibur Lode cycle ergometer (Lode BV, Groningen, Netherlands) was applied. The participant wore his or her own sports shoes and the pedals were mounted with toe-clips. Power output during one-legged pedaling corresponded to body mass times 1.0. Power output during two-legged pedaling was twice as high. It applies to both knee extension exercise and pedaling that frequency was calculated as an average across the last $2 \mathrm{~min}$ of a bout.

Heart rate was measured by a Polar F6 (Electro Oy, Kempele, Finland) every 30th s during each bout and an average value was subsequently calculated across each bout. Rate of perceived exertion (RPE) was indicated by the participant immediately after the bout. For this, a 15-grade Borg RPE scale with scores from 6-20 and corresponding subjective ratings from "Very light" ( 9 on the scale) to "Maximal exertion" (20 on the scale) was applied (Borg, 1970).

The specific outcome measures of the present study were pedaling and knee extension frequencies, power output, heart rate, and RPE.

\section{Statistics}

Pearson product-moment correlation coefficients ( $R$-values) were applied for investigation of the correlation between movement frequencies. Linear regressions were performed for associations presented in Figure 1. Paired-samples $t$-tests were applied for evaluations of potential differences in average values. The ratings based on the size of the $R$-values apply to both positive and negative correlations as follows: $\leq 0.25$ is considered weak, $0.26-0.50$ is moderate, $0.51-0.75$ is fair, and $\geq 0.76$ is high (Berg and Latin, 2008). The $R$-values were tested for significance, i.e., the probability of obtaining the correlation coefficient by chance. ${ }^{1}$ Statistics were calculated in Excel 2010 (Microsoft Corporation, WA, USA). Data are presented as average $\pm \mathrm{SD}$, unless otherwise indicated. Statistical significance was set at $p<0.05$.

\section{RESULTS}

\section{Power Output}

During the one- and two-legged knee extension exercise, power output was $19 \pm 5$ and $37 \pm 9 \mathrm{~W}$, respectively. During one- and two-legged cycling, it amounted to $68 \pm 11$ and $137 \pm 22 \mathrm{~W}$, respectively.

\footnotetext{
${ }^{1}$ http://vassarstats.net/tabs.html
}

\section{Heart Rate and RPE}

Heart rate values were in general low to moderate, which confirmed the submaximal character of the performed activities (Table 1). By estimating maximal heart rate as $208-0.7 \times$ age (Tanaka et al., 2001), average relative heart rates amounted to $68 \%$ of maximum, or less. In line with that, RPE values were in general low to moderate. Thus, the highest average score amounted to 12.6 , which indicated perceived exertion between "Fairly light" and "Somewhat hard", based on the applied Borg RPE scale (Table 1).

\section{Movement Frequencies}

Freely chosen knee extension frequencies ranged from 15-68 rpm across all participants and all three knee extension exercise activities. There was a high correlation between frequencies generated with the dominant leg and the nondominant leg (Figure $\mathbf{1 A}$ and Table 2). Further, there were high correlations between frequencies performed with each of the two legs, separately, and the frequency performed in two-legged knee extension exercise (Figure 1B and Table 2).

For comparison, freely chosen pedaling frequencies ranged from 30-97 rpm across all participants and all three pedaling activities. There was a fair correlation between pedaling frequencies performed with the dominant leg and the nondominant leg (Figure 1C and Table 2). Further, there were fair to high correlations between pedaling frequencies performed with each of the two legs, separately, and the frequency performed in two-legged pedaling (Figure 1D and Table 2). It applies to all $R$-values from correlations between pedaling frequencies $(0.71-0.79)$ that they were lower than the $R$-values from correlations between knee extension frequencies (0.94-0.99; Table 2).

Pedaling frequencies performed in one-legged pedaling with the nondominant leg showed fair correlations ( $R$-values between 0.56 and 0.69 ) with movement frequencies in all knee extension exercise activities (Table 2). But otherwise, there were no significant correlations between pedaling frequencies and knee extension frequencies.

\section{DISCUSSION}

The present study supports the previous finding that freely chosen movement frequency is considerably individual in voluntary stereotyped rhythmic leg movement such as in pedaling (Sarre et al., 2003). In addition to the individual character, such a freely chosen movement frequency appears to be consistent within individuals. As an example, it has been reported that freely chosen pedaling frequency is highly reliable across bouts, within a test session (Hansen et al., 2002), as well as across days (Hansen and Ohnstad, 2008). Of particular note is that the freely chosen knee extension frequency in the present study ranged considerably. Thus, the participant with the highest knee extension frequency worked at an around 350\% higher frequency than the participant with the lowest frequency. That is considerably more than what has been observed for other stereotyped rhythmic movements of e.g., finger tapping (approx. 205\%) and two-legged pedaling (approx. 183\%; 

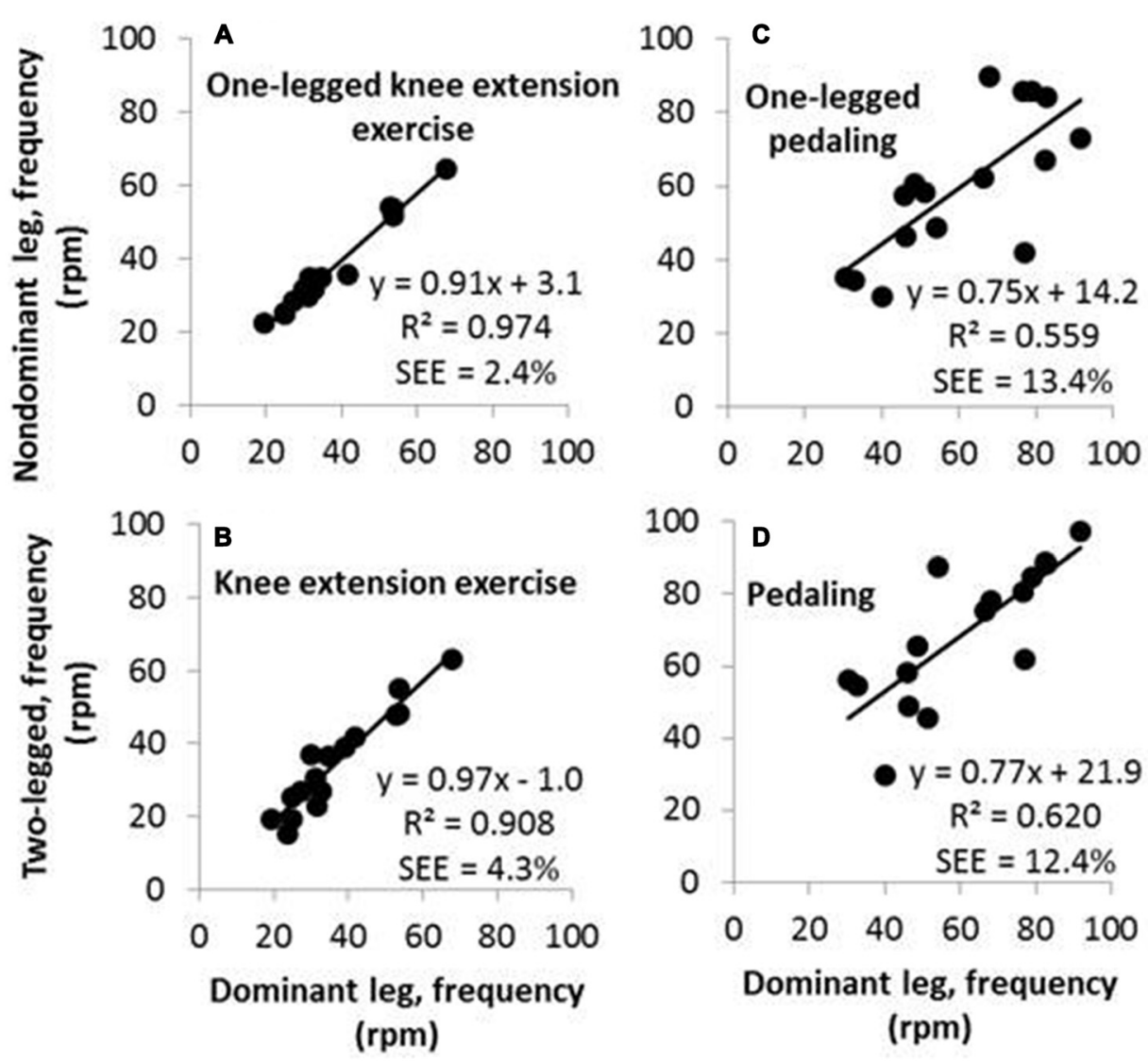

FIGURE 1 | Upper panels contain scatterplots of movement frequencies of the nondominant leg as a function of movement frequencies of the dominant leg in the relatively simple single-joint activity of one-legged knee extension exercise (A) and in the more complex multi-joint activity of one-legged pedaling (C). Lower panels contain scatterplots of movement frequencies of two legs as a function of frequencies of the dominant leg in knee extension exercise (B) and in pedaling (D)

Hansen and Ohnstad, 2008). The reason is unknown and must be investigated in future studies, but the finding is not considered to affect the overall interpretation of the present results.

The present fair to high correlations between movement frequencies generated separately by each of the legs during pedaling and knee extension exercise, respectively, could be interpreted as support of the working hypothesis that the two legs' CPGs share a common frequency generator.

TABLE 1 | Heart rate and RPE presented as average \pm SD.

\begin{tabular}{lcc}
\hline Activity & $\begin{array}{c}\text { Heart rate } \\
\text { (beats per min) }\end{array}$ & $\begin{array}{c}\text { RPE } \\
\text { (Borg scale value) }\end{array}$ \\
\hline Knee extension exercise, one-legged (ND) & $86 \pm 25$ & $8.6 \pm 2.8$ \\
Knee extension exercise, one-legged (D) & $86 \pm 23$ & $8.9 \pm 2.1$ \\
Knee extension exercise, two-legged & $89 \pm 26$ & $8.8 \pm 2.2$ \\
Pedaling, one-legged (ND) & $120 \pm 15$ & $12.3 \pm 2.2$ \\
Pedaling, one-legged (D) & $122 \pm 17$ & $12.4 \pm 2.3$ \\
Pedaling, two-legged & $138 \pm 16^{*}$ & $12.6 \pm 2.4$ \\
\hline
\end{tabular}

$N D$, nondominant; $D$, dominant. ${ }^{*}$ Different from both one-legged pedaling activities ( $p=0.00001$ and 0.0003).
Or alternatively, that separate frequency generators of each of the legs are attuned via interneurons. That hypothesis is illustrated in Figure 2. Existence of separate CPGs for each leg, and the possibility of activating them separately or in combination, has been suggested previously based on studies of leg movements

TABLE 2 | $R$-values from correlations between movement frequencies in the six applied activities (1-6)

\begin{tabular}{|c|c|c|c|c|c|c|}
\hline Activity & 1 & 2 & 3 & 4 & 5 & 6 \\
\hline $1 \mathrm{Knee}$ extension exercise, one-legged (ND) & $i=$ & $\overline{0} . \overline{99}$ & $\overline{0.94}$ & 0.56 & 0.15 & 0.19 \\
\hline 2 Knee extension exercise, one-legged (D) & i & - & 0.95 & 0.67 & 0.30 & 0.26 \\
\hline 3 Knee extension exercise, two-legged & 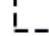 & & & 0.69 & 0.40 & 0.32 \\
\hline 4 Pedaling, one-legged (ND) & & & & $1-$ & $\overline{0.7 \overline{5}}$ & $0 . \overline{71}$ \\
\hline 5 Pedaling, one-legged (D) & & & & i & - & 0.79 \\
\hline 6 Pedaling, two-legged & & & & I & & -- \\
\hline
\end{tabular}

$N D$, nondominant; $D$, dominant. The black dashed rectangle frames $R$-values from correlations between movement frequencies in the relatively simple single-joint activity of knee extension exercise. The gray dashed rectangle frames $R$-values from correlations between movement frequencies in the more complex multi-joint activity of pedaling. Significant $(p<0.05)$ values are indicated by bold. 


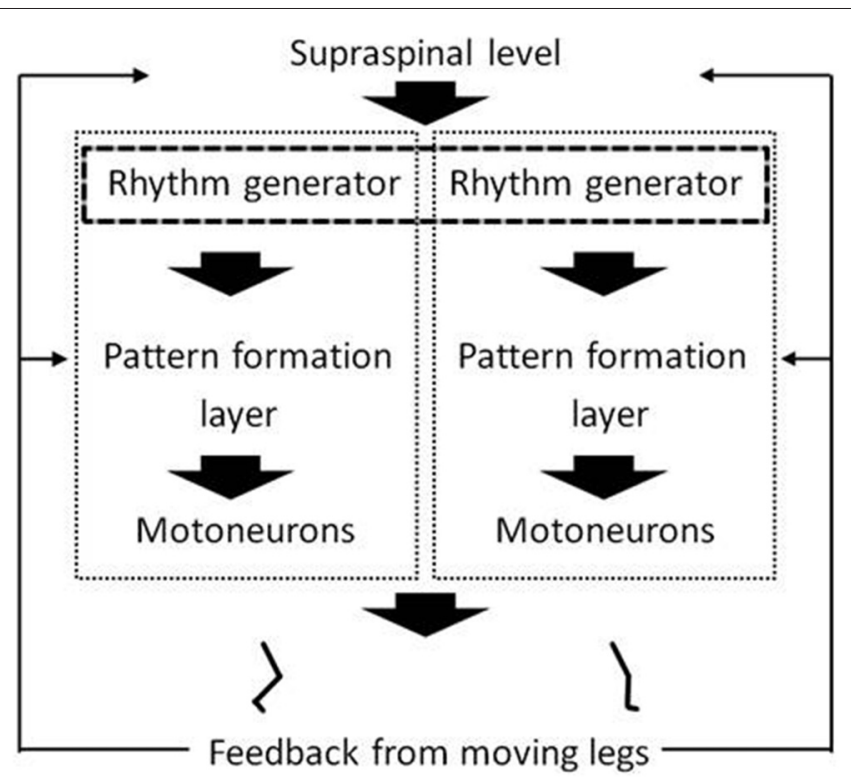

FIGURE 2 | A hypothetical multi-layered organization of rhythm and pattern generators in the spinal cord under influence of descending and sensory input. The figure includes central pattern generators (CPGs) of both legs. The dashed rectangle that frames the rhythm generators is supposed to illustrate the hypothesis that the two legs' CPGs share a common frequency generator, or alternatively, that the separate frequency generators are attuned via interneurons. The gray to black color of the dashed rectangle illustrates the hypothesis that the degree of how common the frequency generation is, is task- or activity-dependent. The figure is inspired by previous work (McCrea and Rybak, 2008; Lacquaniti et al., 2012, 2013).

in completely and incompletely paralyzed patients (Shapkova, 2004). For example, Shapkova (2004) reported unilateral stepping-like movements with spinal cord electrical stimulation of the lumbar enlargement. The present findings obtained on healthy subjects support and add to Shapkova's findings on patients. In addition they supplement research focusing on CPGorganization and -function in lower animals.

A considerable common rhythmogenesis for the two legs could further be suggested based on the present fair to high correlations between frequencies performed in a certain activity with each of the two legs, separately, and the frequency performed in two-legged activity in the same activity.

The activity of pedaling is to a degree characterized by knee extension. Thus, knee extension contributes to characterize the down stroke phase in pedaling where most of the external power is produced. Based on that, it could be hypothesized that generated movement frequencies in pedaling and knee extension exercise might strongly correlate. However, the present study indicated that this did not appear to be the case. Rather, the type of task, or activity, appeared to be of relevance in the present study. Firstly, the common between-leg rhythmogenesis appeared to be strong for knee extension exercise, which may be characterized as relatively simple, single-joint, exercise. For comparison, the common between-leg rhythmogenesis appeared to be weaker for pedaling, which may be characterized as a more complex activity involving multiple joints. An interpretation of this might be that the two legs' CPGs shared a common rhythmogenesis, however, that this characteristic became attenuated during leg movement that involved more segments, joints, and muscles, i.e., in pedaling. Reasons for that could involve differences in degrees of complexity, afferent feedback, and supraspinal input. Another reason that type of activity appeared to be of relevance is the following. There appeared to be only minor sharing of between-activity rhythmogenesis when comparing knee extension exercise and pedaling. For comparison, it has recently been reported that freely chosen stride frequencies in related activities of walking and running correlated $(R=0.72$, $p<0.001$ ) in a group of 27 recreationally active individuals. However, that at the same time, freely chosen pedaling frequency was not correlated with stride frequency in each of the locomotion types of walking and running (Sardroodian et al., 2015). A joint interpretation of those previous, and the present, results could be that when two activities at a point become adequately different, the sharing of between-activity rhythmogenesis attenuates.

The frequency during one-legged pedaling with the nondominant leg showed fair correlation with frequencies during all three types of knee extension exercise. Interestingly, the same was not the case for one-legged pedaling with the dominant leg. This difference is difficult to explain. But, perhaps it indicates that rhythmogenesis during one legged pedaling with the nondominant leg, in particular, to a degree is "driven" by CPGs responsible for rhythmogenesis during the simpler knee extension exercise.

The present type of investigation has some built-in interpretational challenges. For example, it is not possible to directly measure a gross frequency output from a human CPG with available techniques. Here, we suggest that the observed movement frequencies are net, or resultant, outputs from CPGs, i.e., accounting for influence from in particular afferent feedback and supraspinal input. Regarding the complexity of the applied types of activities, it should be noted that during pedaling activities, there is both a pushing phase in which the foot is pushed forward at the top dead center, a down stroke phase where most of the external power is produced, and an upstroke phase in which the leg is partly lifted (So et al., 2005). The down stroke phase is characterized by extension of the knee. Knee extension exercise is simpler in the way that the power production phase and the upstroke phase are coinciding and performed with the same muscles. Less segments, muscles, and joints are involved in knee extension exercise as compared to pedaling that should qualify the former to be characterized as simpler (DaunGruhn, 2011). For the present study, participants adjusted the cycle ergometer settings themselves. It cannot be ruled out that results would have been altered had e.g., a certain relative seat height been applied. Still, most importantly, seat height was maintained across pedaling conditions for each participant.

In conclusion, fair to high correlations occurred between freely chosen movement frequencies generated separately by each of the legs. This might be interpreted to support 
the working hypothesis that the involved CPGs of the two legs share a common frequency generator or that separate frequency generators of each of the legs are attuned via interneuronal connections. Further, the apparent common rhythmogenesis for the two legs appeared to be stronger for the relatively simple single-joint activity of knee extension exercise as compared to the more complex multi-joint activity of pedaling. Finally, it appeared that the shared aspect of knee extension in the related activity types of knee extension exercise and pedaling was insufficient to cause obvious correlations between generated movement frequencies in the two types of activities.

\section{REFERENCES}

Berg, K. E., and Latin, R. W. (2008). Essentials of Research Methods in Health, Physical Education, Exercise Science and Recreation. Baltimore, MD: Wolters Kluwer.

Borg, G. (1970). Perceived exertion as an indicator of somatic stress. Scand. J. Rehabil. Med. 2, 92-98.

Cappellini, G., Ivanenko, Y. P., Poppele, R. E., and Lacquaniti, F. (2006). Motor patterns in human walking and running. J. Neurophysiol. 95, 3426-3437. doi: $10.1152 /$ jn.00081.2006

Daun-Gruhn, S. (2011). A mathematical modeling study of inter-segmental coordination during stick insect walking. J. Comput. Neurosci. 30, 255-278. doi: 10.1007/s10827-010-0254-3

Dominici, N., Ivanenko, Y. P., Cappellini, G., d'Avella, A., Mondi, V., Cicchese, M., et al. (2011). Locomotor primitives in newborn babies and their development. Science 334, 997-999. doi: 10.1126/science.1210617

Goulding, M. (2009). Circuits controlling vertebrate locomotion: moving in a new direction. Nat. Rev. Neurosci. 10, 507-518. doi: 10.1038/nrn2608

Hallén, J., Saltin, B., and Sejersted, O. M. (1996). K+ balance during exercise and role of beta-adrenergic stimulation. Am. J. Physiol. 270, R1347-R1354.

Hansen, E. A. (2015). On voluntary rhythmic leg movement behaviour and control during pedalling. Acta Physiol. (Oxf.) 214, 1-18. doi: 10.1111/apha.12529

Hansen, E. A., Andersen, J. L., Nielsen, J. S., and Sjøgaard, G. (2002). Muscle fibre type, efficiency and mechanical optima affect freely chosen pedal rate during cycling. Acta Physiol. Scand. 176, 185-194. doi: 10.1046/j.1365-201x. 2002.01032.x

Hansen, E. A., and Ohnstad, A. E. (2008). Evidence for freely chosen pedalling rate during submaximal cycling to be a robust innate voluntary motor rhythm. Exp. Brain Res. 186, 365-373. doi: 10.1007/s00221-007$1240-5$

Hansen, E. A., Voigt, M., Kersting, U. G., and Madeleine, P. (2014). Frequency and pattern of rhythmic leg movement in humans after fatiguing exercises. Motor Control 18, 297-309. doi: 10.1123/mc.2013-0044

Ivanenko, Y. P., Poppele, R. E., and Lacquaniti, F. (2006). Motor control programs and walking. Neuroscientist 12, 339-348. doi: 10.1177/1073858406 287987

Kriellaars, D. J., Brownstone, R. M., Noga, B. R., and Jordan, L. M. (1994). Mechanical entrainment of fictive locomotion in the decerebrate cat. J. Neurophysiol. 71, 2074-2086.

Lacquaniti, F., Ivanenko, Y. P., d'Avella, A., Zelik, K. E., and Zago, M. (2013). Evolutionary and developmental modules. Front. Comput. Neurosci. 7:61. doi: $10.3389 /$ fncom.2013.00061

Lacquaniti, F., Ivanenko, Y. P., and Zago, M. (2012). Patterned control of human locomotion. J. Physiol. 590, 2189-2199. doi: 10.1113/jphysiol.2011. 215137

McCrea, D. A., and Rybak, I. A. (2008). Organization of mammalian locomotor rhythm and pattern generation. Brain Res. Rev. 57, 134-146. doi: 10.1016/j. brainresrev.2007.08.006

Minassian, K., Persy, I., Rattay, F., Pinter, M. M., Kern, H., and Dimitrijevic, M. R. (2007). Human lumbar cord circuitries can be activated by extrinsic tonic input

\section{AUTHOR CONTRIBUTIONS}

EAH had the initial idea for the study. All authors contributed to the planning of the study design. JS, MH and HW performed the data collection. All authors contributed on the data analyses and the intepretation of the results. EAH completed the first draft of the article. All authors have participated in revising of draft to finalize the manuscript.

\section{ACKNOWLEDGMENTS}

Participants are thanked for their enthusiastic participation.

to generate locomotor-like activity. Hum. Mov. Sci. 26, 275-295. doi: 10.1016/j. humov.2007.01.005

Perret, C., and Cabelguen, J.-M. (1980). Main characteristics of the hindlimb locomotor cycle in the decorticate cat with special reference to bifunctional muscles. Brain Res. 187, 333-352. doi: 10.1016/0006-8993(80)90207-3

Prochazka, A., and Ellaway, P. (2012). Sensory systems in the control of movement. Compr. Physiol. 2, 2615-2627. doi: 10.1002/cphy.c100086

Sardroodian, M., Madeleine, P., Voigt, M., and Hansen, E. A. (2015). Freely chosen stride frequencies during walking and running are not correlated with freely chosen pedalling frequency and are insensitive to strength training. Gait Posture 42, 60-64. doi: 10.1016/j.gaitpost.2015.04.003

Sarre, G., Lepers, R., Maffiuletti, N., Millet, G., and Martin, A. (2003). Influence of cycling cadence on neuromuscular activity of the knee extensors in humans. Eur. J. Appl. Physiol. 88, 476-479. doi: 10.1007/s00421-0020738-6

Schlinger, H. D. (2015). Behavior analysis and behavioral neuroscience. Front. Hum. Neurosci. 9:210. doi: 10.3389/fnhum.2015.00210

Shapkova, E. Y. (2004). "Spinal locomotor capability revealed by electrical stimulation of the lumber enlargement in paraplegic patients," in Progress in Motor Control, eds M. Latash and M. Levin (Champaign, IL: Human Kinetics), 253-289.

So, R. C. H., Ng, J. K.-F., and Ng, G. Y. F. (2005). Muscle recruitment pattern in cycling: a review. Phys. Ther. Sport 6, 89-96. doi: 10.1016/j.ptsp.2005.02.004

Tanaka, H., Monahan, K. D., and Seals, D. R. (2001). Age-predicted maximal heart rate revisited. J. Am. Coll. Cardiol. 37, 153-156. doi: 10.1016/s07351097(00)01054-8

Zehr, E. P. (2005). Neural control of rhythmic human movement: the common core hypothesis. Exerc. Sport Sci. Rev. 33, 54-60.

Zehr, E. P., Balter, J. E., Ferris, D. P., Hundza, S. R., Loadman, P. M., and Stoloff, R. H. (2007). Neural regulation of rhythmic arm and leg movement is conserved across human locomotor tasks. J. Physiol. 582, 209-227. doi: 10.1113/jphysiol. 2007.133843

Zehr, E. P., and Duysens, J. (2004). Regulation of arm and leg movement during human locomotion. Neuroscientist 10, 347-361. doi: 10 $1177 / 1073858404264680$

Conflict of Interest Statement: The authors declare that the research was conducted in the absence of any commercial or financial relationships that could be construed as a potential conflict of interest.

The Review Editor Dr. Pedro Figueiredo declares that, despite being affiliated with the same institution as the Associate Editor Dr. Jae Kun Shim, the review process was handled objectively.

Copyright (๑) 2016 Stang, Wiig, Hermansen and Hansen. This is an open-access article distributed under the terms of the Creative Commons Attribution License (CC $B Y)$. The use, distribution and reproduction in other forums is permitted, provided the original author(s) or licensor are credited and that the original publication in this journal is cited, in accordance with accepted academic practice. No use, distribution or reproduction is permitted which does not comply with these terms. 